\title{
Capturing Evolving Visit Behavior in Clickstream Data
}

\author{
Wendy W. Moe and Peter S. Fader
}

January 2001

Wendy W. Moe (wendy.moe @bus.utexas.edu) is Assistant Professor of Marketing at the University of Texas at Austin, CBA 7.216, Austin, Texas 78712. Peter S. Fader (fader@wharton.upenn.edu) is Associate Professor of Marketing at the Wharton School of the University of Pennsylvania, 1400 Steinberg-Dietrich Hall, Philadelphia, Pennsylvania 19104. The authors would like to thank WeBI (the Wharton e-Business Initiative) and Media Metrix, Inc., for generously providing the data used in this study as well as Bruce Hardie and Eric Bradlow for their very useful comments and suggestions. This paper stems from Wendy Moe's dissertation work, and she extends special thanks to the other members of her dissertation committee (Barbara Kahn, Don Morrison, and David Schmittlein) and the Marketing Science Institute for supporting this research through the 1999 Alden Clayton Dissertation Award. 


\title{
Capturing Evolving Visit Behavior in Clickstream Data
}

\begin{abstract}
:
Many online retailers monitor visitor traffic as a measure of their stores' success. However, summary measures such as the total number of visits per month provide little insight about individual-level shopping behavior. Additionally, behavior may evolve over time, especially in a changing environment like the Internet. Understanding the nature of this evolution provides valuable knowledge that can influence how a retail store is managed and marketed.

This paper develops an individual-level model for store visiting behavior based on Internet clickstream data. We capture cross-sectional variation in store-visit behavior as well as changes over time as visitors gain experience with the store. That is, as someone makes more visits to a site, her latent rate of visit may increase, decrease, or remain unchanged as in the case of static, mature markets. So as the composition of the customer population changes (e.g., as customers mature or as large numbers of new and inexperienced Internet shoppers enter the market), the overall degree of visitor heterogeneity that each store faces may shift.

We also examine the relationship between visiting frequency and purchasing propensity. Previous studies suggest that customers who shop frequently may be more likely to make a purchase on any given shopping occasion. As a result, frequent shoppers often comprise the preferred target segment. We find evidence supporting the fact that people who visit a store more frequently are more likely to buy. However, we also show that changes (i.e., evolution) in an individual's visit frequency over time provides further information regarding which customer segments are more likely to buy. Rather than simply targeting all frequent shoppers, our results suggest that a more refined segmentation approach that incorporates how much an individual's behavior is changing could more efficiently identify a profitable target segment.
\end{abstract}

KEYWORDS: Electronic commerce, Clickstream data, Evolving Behavior, Duration models, Heterogeneity, Stochastic models, Nonstationarity 


\section{Introduction}

Ever since the Internet first emerged as a viable medium for commercial purposes, analysts have closely tracked visitor traffic as a principal yardstick to gauge the success of online retail sites. Even after the unprecedented highs and lows that the e-commerce world experienced recently, visit-related measures continue to be among the most widely used metrics by virtually all industry experts, including retail managers, journalists, consultants, and even investors (Demers and Lev 2000).

But the dramatic changes that took place in the year 2000 led to changes in the way that traffic measures are viewed and utilized. In the early days of e-commerce, simple measures of unique visitors served as a proxy for site performance. Most retailers subsequently learned (the hard way, in many cases) that this is not a useful indicator; instead, measures of visitor retention and loyalty have proven to be more closely linked to the health of their businesses. Accordingly, measures such as visits per visitor have gained more prominence and are now included in the ecommerce "scorecards" that firms such as Media Metrix and Nielsen/NetRatings routinely publish.

In Figure 1 we show the distribution of the number of visits per visitor for two leading ecommerce sites, Amazon.com and CDNOW, over a period of eight months in 1998. This aggregate "snapshot" shows some clear, systematic differences across the customer base, e.g., the high proportion of infrequent visitors, a pattern very similar to what has been observed for a variety of offline behavioral patterns. But unlike many well-established, relatively mature markets, online visit patterns are still in a state of transition. The static summary of differences 
in visit behavior across customers shown in Figure 1 may be masking some significant changes over time at the individual level. When we acknowledge the existence of both of these sources of heterogeneity (i.e., differences across individuals and over time), it can become difficult - yet critically important - to separate them out from each other.

Figure 1. Histograms of Visits per Visitor

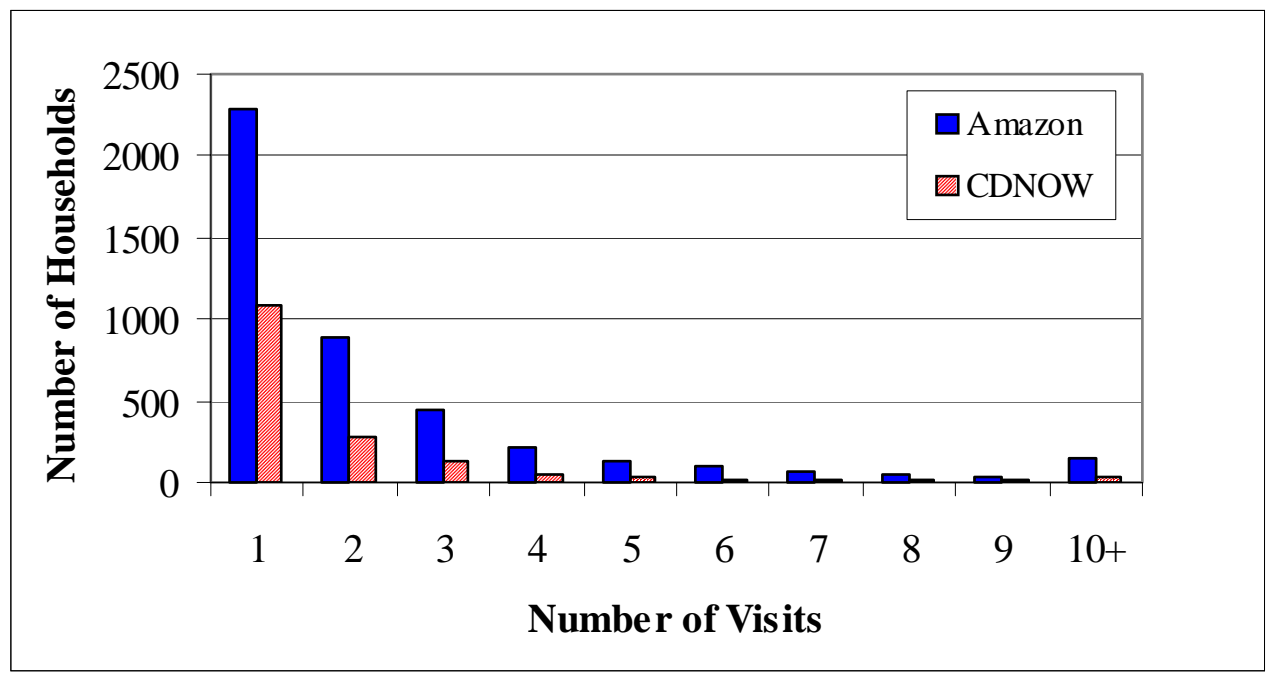

As a specific example, Table 1 provides an aggregate summary of the dynamics in visit patterns underlying the histograms shown in Figure 1. For both Amazon and CDNOW, not only do we see growth in the total number of visits over time, but there also appears to be an increase in the number of visits per visitor. On the surface, these aggregate measures seem like great news for the store managers. However, these numbers may be misleading as the customer base is changing with the influx of new visitors (perhaps with relatively high visit rates) and the exit of more experienced users, potentially masking the true visit dynamics that exist. 
Table 1. Summary of Visit Data Over Time

\begin{tabular}{|l||c|c||c|c|}
\hline \multicolumn{1}{|c||}{} & \multicolumn{2}{c||}{ Amazon } & \multicolumn{2}{c|}{ CDNOW } \\
\cline { 2 - 4 } & Months 1-4 & Months 5-8 & Months 1-4 & Months 5-8 \\
\hline $\begin{array}{l}\text { Total Number } \\
\text { of Visits }\end{array}$ & 5402 & 5899 & 1729 & 1890 \\
\hline $\begin{array}{l}\text { Number of } \\
\text { Unique Visitors }\end{array}$ & 2693 & 2717 & 988 & 920 \\
\hline Visits/Visitor & 2.01 & 2.17 & 1.75 & 2.05 \\
\hline
\end{tabular}

Figure 2 provides a deeper view of visit trends with a histogram of the average intervisit time and how it varies across visit cycles. Again, deceptively, this view of the data indicates that as a customer repeatedly visits a site, intervisit times decrease slightly, i.e., visits become more frequent over time, consistent with the data presented in Table 1. However, this is also an inaccurate view of the actual trends in visitor behavior. A major drawback of this chart is the selection bias that directly causes the downward trend in intervisit times. That is, those shoppers who have had the opportunity to make more visits in a fixed period of time will, by definition, have shorter average intervisit times than those shoppers for whom we could only observe a small number of visits in the same time interval. These frequent shoppers are the ones that dominate the right side of the histogram shown in Figure 1, and as such, the apparent downward trend in intervisit times across visit cycles is unavoidable and highly nonrepresentative of the full set of visitors to each site. Even the first column of Figure 2 (i.e., average time to first repeat) is misleading since it ignores the large fraction of one-time-only visitors to each site. 
Figure 2. Average Intervisit Time Across Visit Cycles

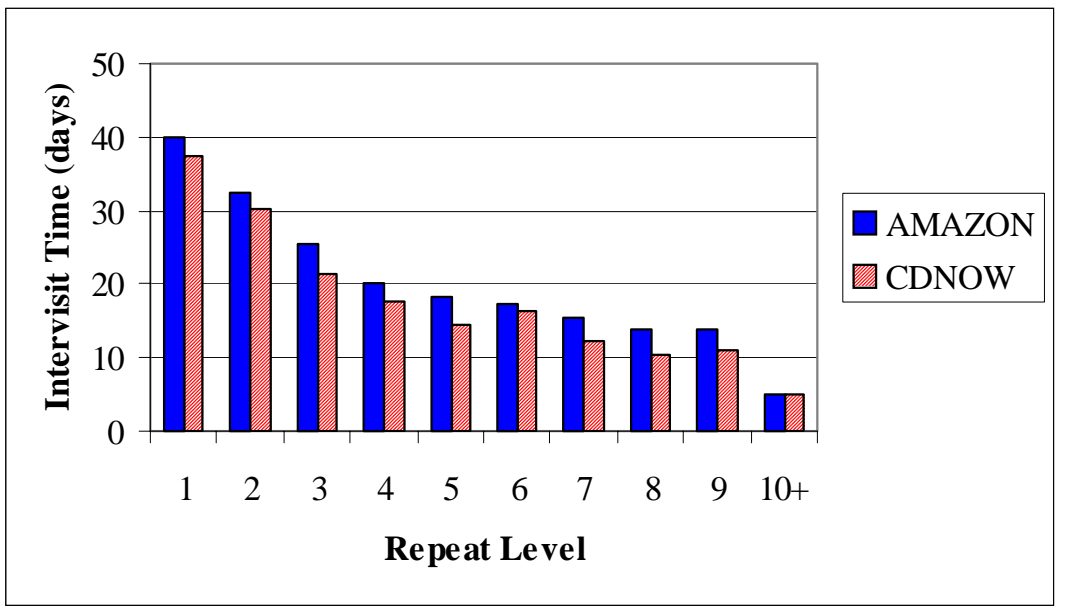

In short, any attempt to summarize intervisit times directly from observed data will be unable to provide accurate estimates of the true, underlying rates of repeat behavior. Likewise, any attempt to uncover dynamics in visit rates by splitting the sample into groups of "early" versus "late" visits will run into similar problems. The only way to overcome these selection biases while still ensuring the representativeness of the entire set of visitors is to use a well-specified individuallevel model (with suitable assumptions about heterogeneity) to obtain inferences about differences in visit patterns across people and over time.

Therefore, one objective of this paper is to develop a probabilistic model that carefully sorts out all of these issues. An important (and unique) aspect of this model is the manner in which we allow for evolving behavior in visitor traffic. Traditional stochastic models of purchasing behavior assume that purchase rates are unchanging over time (e.g., Morrison and Schmittlein 1988). When these models are tested in stable and mature markets, such an assumption may indeed hold. But many new markets go through a state of flux for quite some time (Bronnenberg, Mahajan, and Vanhonacker 2000). In other words, an individual's shopping 
behavior often changes as she continually adapts to a new environment. The model presented in this paper will relax the usual assumption of stationarity. More specifically, the evolutionary component of the model allows shoppers to return to the store either more or less frequently as they gain experience, while also accommodating customer attrition for those individuals who never return after one or more initial visits to the site.

From our evolving visit model, we can estimate how likely (and when) a given shopper will return to the store as she gains experience with a website. Do intervisit times tend to speed up or slow down over a person's history, and how do these changes vary across people? Answers to these questions will give us the ability to forecast future store visits in order to better anticipate and manage website traffic. We will show that our evolving model of visiting behavior forecasts traffic patterns significantly better than an equivalent static model. Additionally, the evolutionary component of the model will offer useful diagnostics that will help shed light on other aspects of online shopping behavior. For example, are frequent shoppers necessarily more valuable segments to target?

Though Internet clickstream data is rich with behavioral information such as duration of visits, number of page views, characteristics of items viewed, etc., we examine only the timing and frequency of store visits, as understanding visitor traffic at this level is an important managerial issue in itself. However, despite the limited data that we use, we find that simple visiting rates (and trends in these rates) are strong indicators of an individual's buying propensity. As we better describe customers in terms of their visiting behavior, we relate visiting frequency to purchasing propensity. Previous studies suggest that people who shop frequently may be more 
likely to make a purchase on any given shopping occasion (Bellenger, Robertson, and Hirschman 1978, Jarboe and McDaniel 1987, Roy 1994). As a result, frequent shoppers are often the preferred target segment. Our clickstream analysis strongly confirms this hypothesis, and then extends it by showing that changes (i.e., evolution) in an individual's visit frequency over time provide even better information regarding which customers (and customer segments) are more likely to buy. Rather than simply targeting all frequent shoppers, our results suggest that a more refined segmentation approach that incorporates how much an individual's behavior is changing can more efficiently identify profitable customers for targeting purposes.

In the next two sections, we develop the model and address some of the key estimation issues that arise from the model. We then describe the clickstream data that we will be using. In $\S 5$, we will present the results of the model when applied to two leading online retailers and briefly discuss some of the managerial implications of the results. We validate the model by demonstrating its forecasting ability over a four-month holdout period. Finally, in $\S 6$, we will illustrate how purchasing behavior varies across shoppers as a function of their latent visit rates as well as changes in these rates over time.

\section{Model Development}

To understand the overall pattern of store visits, let us imagine that each shopper tends to return to a store at a latent rate inherent to that individual. When that individual will visit the store next is driven largely by this rate of visit. Additionally, since customers are heterogeneous, this rate of visit varies from person to person. Some people may visit the store fairly frequently while others may not. But in addition to varying rates of visit across individuals, behavior may also 
change over time for a given individual. As shoppers mature, perhaps as a result of increased knowledge and experience, their behavior may evolve thereby changing their rates of visit over time.

To capture the processes described above, our model has three main components:

(1) A timing process governing an individual's rate of visiting,

(2) A heterogeneity distribution that accommodates differences across people, and

(3) An evolutionary process that allows a given individual's underlying visit rate to change from one visit to the next.

\section{Timing process with heterogeneity}

As an appropriately robust starting point, repeat visit behavior can be modeled as an exponentialgamma (EG) timing process. That is, each individual's intervisit time is assumed to be exponentially distributed governed by a rate, $\lambda_{i}{ }^{1}$ Furthermore, these individual rates of visit vary across the population. This heterogeneity can be captured by a gamma distribution with shape parameter, $r$, and scale parameter, $\alpha$. These distributions are given by the following two densities:

$$
f\left(t_{i j} ; \lambda_{i}\right)=\lambda_{i} e^{-\lambda_{i}\left(t_{i j}-t_{h(j-1)}\right)} \quad \text { and } \quad g\left(\lambda_{i} ; r, \alpha\right)=\frac{\lambda_{i}^{r-1} \alpha^{r} e^{-\alpha \lambda_{i}}}{\Gamma(r)}
$$

\footnotetext{
${ }^{1}$ Alternative timing distributions, such as the Erlang-2, were also examined but performed consistently worse than the exponential.
} 
where $\lambda_{i}$ is individual $i$ 's latent rate of visit, $t_{i j}$ is the day when the $j^{\text {th }}$ repeat visit occurred, and $t_{i 0}$ is the day of their first observed visit. For a single visit occasion, this leads to the following familiar exponential-gamma mixture model:

$$
f\left(t_{i j} ; r, \alpha\right)=\int_{0}^{\infty} f\left(t_{i j} ; \lambda_{i}\right) \cdot g\left(\lambda_{i} ; r, \alpha\right) d \lambda=\frac{r}{\alpha}\left(\frac{\alpha}{\alpha+\left(t_{i j}-t_{i(j-1)}\right)}\right)^{r+1}
$$

While the exponential-gamma may be an excellent benchmark model, it fails to capture nonstationarity over time. To account for nonstationarity, extensions of this model are described next.

\section{Evolving Behavior}

In the relatively new and fast-paced Internet environment, it is particularly important to address the issue of evolving behavior as people are continually updating their behavior, and web retailers must adapt to keep up with their customers. For example, studies have shown that as a shopper's knowledge and familiarity increase over time, the extent of search she undertakes may change, either increasing or decreasing depending on the situation (see Alba and Hutchinson 1987, Johnson and Russo 1984, Park, Iyer, and Smith 1989). Typically, increased store knowledge and familiarity lead to more efficient search behavior. This increased shopping efficiency may have one of two effects on future store visiting behavior. One, the amount of explicit search required to make a purchase decision decreases as shoppers have more internal knowledge from which to draw (Bettman 1979, Johnson and Russo 1984, Park, Iyer, and Smith 1989). This may lead to less frequent store visits as the customer adapts to the shopping situation and the novelty of the site wears off. On the other hand, Johnson and Russo (1984) have also 
shown that more knowledgeable shoppers will search more since they can search more efficiently. As a result, store visits may become more frequent over time for an individual shopper.

Though these studies suggest that search and store visiting behavior may evolve as a function of experience, they are inconclusive in identifying the direction of this evolution. Therefore, our model is a descriptive one that captures and characterizes any evolution that may exist. We develop a flexible model that will accommodate varying magnitudes and directions of the behavioral change and offer a method to characterize the nature of this evolution.

Researchers in marketing have used several different mechanisms to introduce these time-varying effects into the traditional stochastic modeling framework. For instance, Sabavala and Morrison (1981) incorporated nonstationarity by introducing a renewal process into a probability mixture model in accordance with the "dynamic inference" framework first set out by Howard (1965). Sabavala and Morrison applied this model to explain patterns of advertising media exposure over time; further applications of a similar type of renewal-process approach can be seen in Fader and Lattin 1993 as well as Fader and Hardie 1999.

While these renewal models provide one mechanism to introduce nonstationarity into a timing process, they do not offer any appealing way to capture the type of evolutionary process that we have described above. The aforementioned renewal models operate under the assumption that each shopper probabilistically discards their old rate parameter and draws an entirely new one from the original heterogeneity distribution, independent of previous values. This process allows for drastic changes in an individual's behavior while maintaining the same heterogeneity 
distribution for the population as a whole. While this may be a powerful and effective way to capture large changes over time, it is not consistent with the type of gradual, evolutionary behavioral changes that are likely to occur from visit to visit. Furthermore, in an evolving market environment, it may be incorrect to assume that the overall heterogeneity distribution is not changing over time. The EV model that we propose allows for the population heterogeneity distribution to change as the customers that comprise the population gradually update their visit rates.

Specifically, our behavioral assumption is that customers' underlying rates of visiting are continually and incrementally changing from one visit to the next. As individuals adapt to and gain experience with the new retail environment, they may return to the store at a more frequent rate, a less frequent rate, or perhaps at the same rate for the next visit. By assuming that each individual will update her latent rate, $\lambda_{i}$, after each visit, a very simple way to specify this updating process is as follows:

$$
\lambda_{i(j+1)}=\lambda_{i j} \cdot c
$$

where $\lambda_{i j}$ is the rate associated with individual $i$ 's $j^{\text {th }}$ repeat visit and $c$ is a multiplier that will update this rate from one visit to the next. If the updating multiplier, $c$, equals one, visiting rates are considered unchanging, and the stationary exponential-gamma would remain in effect. But if $c$ is greater than one, shoppers are visiting more frequently as they gain experience, and if $c$ is less than one, shoppers are visiting less frequently as they gain experience. 
However, using a constant multiplier to update the individual $\lambda$ 's would be a very restrictive (and highly unrealistic) way of modeling evolutionary behavior in a heterogeneous environment. A more general approach is to replace the scalar multiplier, $c$, with a random variable $c_{i j}$ in order to acknowledge that these updates can vary over time and across people. Each individual visit will lead to an update that may increase, decrease, or retain the previous rate of visit, depending on the stochastic nature of the updating multiplier.

To generalize (3) in this manner, we assume that these probabilistic multipliers, $c_{i j}$, arise from a gamma distribution, common across individuals and visits, with shape parameter $s$ and scale parameter $\beta$. We choose the gamma distribution to describe the updating multipllier for the same reasons why we used it to describe the heterogeneity in $\lambda$ - it is a very flexible distribution that accommodates a variety of shapes ${ }^{2}$. This gamma distribution essentially describes the nature of the behavioral evolution faced by a given store. The updated $\lambda_{i(j+1)}$ then becomes a product of two independent gamma-distributed random variables ${ }^{3}$ : the previous rate, $\lambda_{i j}$, and the multiplier, $c_{i j}$. The overall model, therefore, uses four parameters to simultaneously capture cross-sectional heterogeneity and evolving visiting behavior: two parameters ( $r$ and $\alpha$ ) govern the gamma distribution that describes the initial heterogeneity in visiting rates, and another two parameters $(s$ and $\beta$ ) govern the gamma distribution that describes the updating process. This is the entire model specification.

\footnotetext{
${ }^{2}$ A cursory look at the ratio of intervisit times for a given level of repeat to the intervisit times of the last visit cycle suggests that the gamma distribution is an adequate descriptor of visit-to-visit changes.

${ }^{3}$ We explored the potential interdependence of $\lambda_{i j}$ and $c_{i j}$ by modeling the shape parameter of the $c_{i j}$ distribution as a function of $E\left[\lambda_{i j}\right]$ but found that this model specification becomes rather cumbersome and does not perform substantially better than one that assumes independence.
} 
Regardless of whether the multiplier is increasing $\left(c_{i j}>1\right)$ or decreasing $\left(c_{i j}<1\right)$ a particular visit rate at a particular point in time, we expect that an individual's value of $\lambda$ will evolve relatively slowly over time. This suggests that the updating gamma distribution, $u\left(c_{i j} ; s, \beta\right)$, should have a mean fairly close to 1.0 but should also allow for more extreme increases or decreases in $\lambda$ at any given update opportunity. The spread of this updating distribution is directly tied to the magnitude of the $s$ and $\beta$ parameters. As both of these parameters become large, the distribution degenerates towards a spike located at s/ $\beta$. Taken to the extreme (i.e., $s$ and $\beta$ get extremely large), this model would then collapse into the deterministic updating model (3) with $\mathrm{c}=\mathrm{s} / \beta$.

Finally, another interesting characteristic of the updating distribution is that it allows for customer attrition, since the gamma distribution can yield a draw of $c_{i j}$ extremely close to 0 . When this situation arises, the customer effectively drops out and is unlikely to return to the site. Such attrition may be very common for websites and has been the centerpiece of other types of models in this general methodological area (Reinartz and Kumar 2000; Schmittlein, Morrison, and Colombo 1989). The fact that we can accommodate attrition in such a simple, natural manner is an appealing aspect of the proposed modeling approach.

\section{Likelihood Specification}

When estimating the ordinary (stationary) exponential-gamma model, there are two ways of obtaining the likelihood function for a given individual. The usual approach is to specify the individual-level likelihood function, conditional on that person's (unobserved) value of $\lambda_{i}$. This likelihood is the product of $J_{i}$ exponential timing terms, where $J_{i}$ is the number of repeat visits 
made by panelist $i$, plus an additional term to account for the right-censoring that occurs between that customer's last arrival and the end of the observed calibration period (at time $T$ ):

$$
L_{i} \mid \lambda_{i}=\lambda_{i} e^{-\lambda_{i}\left(t_{i 1}-t_{i 0}\right)} \cdot \lambda_{i} e^{-\lambda_{i}\left(t_{i 2}-t_{i 1}\right)} \cdot \ldots \cdot \lambda_{i} e^{-\lambda_{i}\left(t_{i J_{i}}-t_{i\left(J_{i}-1\right)}\right)} \cdot e^{-\lambda_{i}\left(T-t_{i J_{i}}\right)}
$$

To get the unconditional likelihood we then integrate across all possible values of $\lambda$, using the gamma distribution as a weighting function:

$$
L_{i}\left|r, \alpha=\int_{0}^{\infty} L_{i}\right| \lambda_{i} \cdot \operatorname{gamma}\left(\lambda_{i} ; r, \alpha\right) d \lambda_{i}
$$

where gamma $\left(\lambda_{i} ; r, \alpha\right)$ denotes the gamma distribution as shown in (1). This yields the usual exponential-gamma likelihood, which can be multiplied across the $N$ panelists to get the overall likelihood for parameter estimation purposes:

$$
L=\prod_{i=1}^{N} \frac{\Gamma\left(r+J_{i}\right)}{\Gamma(r)}\left(\frac{\alpha}{\alpha+T-t_{i 0}}\right)^{r}\left(\frac{1}{\alpha+T-t_{i 0}}\right)^{J_{i}}
$$

An alternative path that leads to the same result is to perform the gamma integration separately for each of the $J_{i}+1$ exponential terms, and then multiply them together at the end. This involves the use of Bayes Theorem to refine our "guess" about each individual's value of $\lambda_{i}$ after each arrival occurs. Specifically, it is easy to show that if someone's first repeat visit occurs at time $t_{i j}$, then:

$$
g\left(\lambda_{i 2} \mid \text { arrival at } t_{i l}\right)=\operatorname{gamma}\left(r+1, \alpha+t_{i l}-t_{i 0}\right)
$$

The gamma distribution governing the rate of visit for subsequent arrivals follows: 


$$
g\left(\lambda_{i(j+1)} \mid \text { arrival at } t_{i j}\right)=\operatorname{gamma}\left(r+j, \alpha+t_{i j}-t_{i 0}\right)
$$

Using this logic, we can re-express the likelihood as the product of separate EG terms

$$
\begin{aligned}
& L=\prod_{i=1}^{N} \prod_{j=1}^{J_{i}}\left(\frac{r+j+1}{\alpha+t_{i(j-1)}-t_{i 0}}\right)\left(\frac{\alpha+t_{i(j-1)}-t_{i 0}}{\alpha+t_{i j}-t_{i 0}}\right)^{r+j} \cdot S\left(T-t_{i J_{i}}\right) \\
& \text { where } S\left(T-t_{i J_{i}}\right)=\left(\frac{\alpha+t_{i J_{i}}-t_{i 0}}{\alpha+T-t_{i 0}}\right)^{r+J_{i}}
\end{aligned}
$$

which collapses into the same expression as (6).

When we introduce the nonstationary updating distribution, the multipliers $\left(c_{i j}\right)$ change the value of $\lambda_{i}$ from visit to visit, thereby requiring us to use the sequential approach given in (9) to derive the complete likelihood function. We need to capture two forms of updating after each visit: one due to the usual Bayesian updating process (which is associated with stationary behavior given by (8)) and the other due to the effects of the stochastic evolution process. Therefore, the distribution of visiting rates at each repeat visit level is the product of two gamma distributed random variables - one associated with the updating multiplier and one capturing the previous visiting rate. For the case of panelist $i$ making her $j^{\text {th }}$ repeat visit at time $t_{i j}$ :

$$
G\left(\lambda_{i(j+1)} \mid \text { arrival at } t_{i j}\right)=\operatorname{gamma}\left(r+j, \alpha+t_{i j}-t_{i 0}\right) \cdot \operatorname{gamma}(s, \beta)
$$

One issue with this approach is that the product of two gamma random variables does not lend itself to a tractable analytic solution. However, there is an established result (see, e.g., Kendall and Stuart 1977, p. 248) suggesting that the product of two gamma distributed random variables 
can be approximated by yet another gamma distribution, obtained by multiplying the first two moments about the origins of the original distributions:

$$
\begin{aligned}
& m_{1}^{\left(\lambda_{i(j+1)}\right)}= m_{1}{ }^{\left(\lambda_{i j}\right)} \times m_{1}{ }^{\left(c_{i j}\right)} \\
& \text { and } \\
& m_{2}^{\left(\lambda_{i(j+1)}\right)}=m_{2}{ }^{\left(\lambda_{i j}\right)} \times m_{2}{ }^{\left(c_{i j}\right)}
\end{aligned}
$$

As shown in Appendix A, this moment-matching approximation, used in conjunction with Bayesian updating, allows us to recover the updated gamma parameters that determine the rate of visit, $\lambda_{i j}$, for panelist $i$ 's $j^{\text {th }}$ repeat visit as follows:

$$
\begin{aligned}
& r(i, j+1)=\frac{\lfloor r(i, j)+1\rfloor \cdot s}{[r(i, j)+2\rfloor \cdot(s+1)-\lfloor r(i, j)+1] \cdot s} \\
& \alpha(i, j+1)=\frac{\left\lfloor\alpha(i, j)+t_{i j}-t_{i(j-1)}\right\rfloor \cdot \beta}{[r(i, j)+2] \cdot(s+1)-[r(i, j)+1] \cdot s}
\end{aligned}
$$

where $r(i, 1)$ and $\alpha(i, 1)$ are equal to the initial values of $r$ and $\alpha$ as estimated by maximizing the likelihood function specified in (8).

We performed 20 separate simulations to verify the accuracy of using such a moment-matching approximation. In each simulation, we first generated 1000 random draws from a gamma distribution with randomly determined shape and scale parameters to represent initial $\lambda$ values. Then, a matrix of updating multipliers were also simulated for a series of five updates (i.e., five future repeat visits). Each 1000x5 matrix was generated by taking draws from a gamma distribution, again with randomly determined shape and scale parameters, where columns one 
through five represented the updates after one to five visits. The updated $\lambda$ series after five repeat visits was calculated using two methods (1) direct (numerical) multiplication of the 1000 initial $\lambda$ 's and the five updating series or (2) randomly drawing 1000 values from the distribution resulting from the moment-matching approximation across all five updates. A KolmogorovSmirnov test of fit indicated that, for each of the 20 simulations, the distribution of values resulting from the moment-matching approximation is not significantly different from that resulting from the direct multiplication of these random variables. Therefore, we are confident that the moment-matching approximation accurately captures the gamma distributed updating process we wish to model.

After incorporating the evolution process into our model, the likelihood function to be maximized follows:

$$
L=\prod_{i=1}^{N} \prod_{j=1}^{J_{i}}\left(\frac{r(i, j)}{\alpha(i, j)}\right)\left(\frac{\alpha(i, j)}{\alpha(i, j)+t_{i j}-t_{i(j-1)}}\right)^{r(i, j)+1} \cdot S\left(T-t_{i J_{i}}\right)
$$

where $r(i, j)$ and $\alpha(i, j)$ are defined in equations (12) and (13) while the survival function, $S\left(T-t_{i j}\right)$, is defined as:

$$
S\left(T-t_{i j}\right)=\left(\frac{\alpha\left(i, J_{i}+1\right)}{\alpha\left(i, J_{i}+1\right)+T-t_{i J_{i}}}\right)^{r\left(i, J_{i}+1\right)}
$$

For the special case in which behavior is not evolving and the nonstationary updating distribution degenerates to a spike at 1.0 (i.e., $s=\beta=\mathrm{M}$, where $\mathrm{M}$ approaches infinity), then this equation collapses down exactly to the ordinary (stationary) exponential-gamma model. 


\section{Data}

We apply the models described in the previous section to clickstream data collected by Media Metrix, Inc. Media Metrix maintains a panel of approximately 10,000 households whose Internet behavior (and in fact, all computer behavior) is recorded, pageview by pageview, over time. Participating households install Media Metrix software on their personal computers. While panelists surf the Internet, the software runs in the background and records the date, time, and duration of each and every page being viewed.

For our purposes, we are interested in the dates of the visits each panelist makes to a given store site. Any session in which the web user views a URL with a particular online store's domain name is considered a visit to that store. To consolidate the data just a bit, we aggregated visits to the daily level. For example, a shopper may leave a store briefly and return later that day. However, this second visit is unlikely to be considered a repeat visit but rather an extension of the first visit. Therefore, if a given panelist were to visit a particular store multiple times in a single calendar day, we would encode that behavior as just one visit for the day when the session began. Since we are interested in the timing and frequency of repeat visits to a store, our dataset describes each panelist as a sequence of days when visits were made. All panelists that have visited the store of interest at least once during the observation period were included in this dataset. We use data from March 1, 1998 to October 31, 1998 for two online store sites Amazon.com and CDNOW.com. Amazon attracted 4,379 unique visitors to its site during this eight-month period totaling 11,301 visits, while CDNOW had 1,670 visitors making 3,619 visits (refer back to Figure 1 and Table 1 for more detailed summaries of the data). 


\section{Model Results}

Before estimating the evolving visit model developed in $\S 3$, we first examine the static exponential-gamma timing model as a benchmark. When the static, two-parameter model is applied to the eight months of Amazon data, we find that the mean rate of visit $(E[\lambda]=r / \alpha)$ is 0.0112. In other words, the expected intervisit time $(1 / \lambda)$ is 89.3 days, which is high, but reasonably consistent with the summary statistics mentioned earlier. But beyond their ability to capture the mean of the heterogeneous visiting process, the model parameters also provide useful information about the nature of the distribution of visit rates across the population. With a shape parameter of 0.483 and a scale parameter of 42.955 , the distribution of visiting rates can be described by the gamma distribution in Figure 3. This distribution has a large proportion of the population with very low rates of visit. The median rate, according to this model, is 0.005 , corresponding to an intervisit time of 200 days. This distribution of rates is very consistent with the observed histogram of visit frequency (Figure 1), suggesting that the stationary EG model provides a very good benchmark model for visit behavior.

Figure 3. Gamma Distribution of Visiting Rates for Stationary EG Model

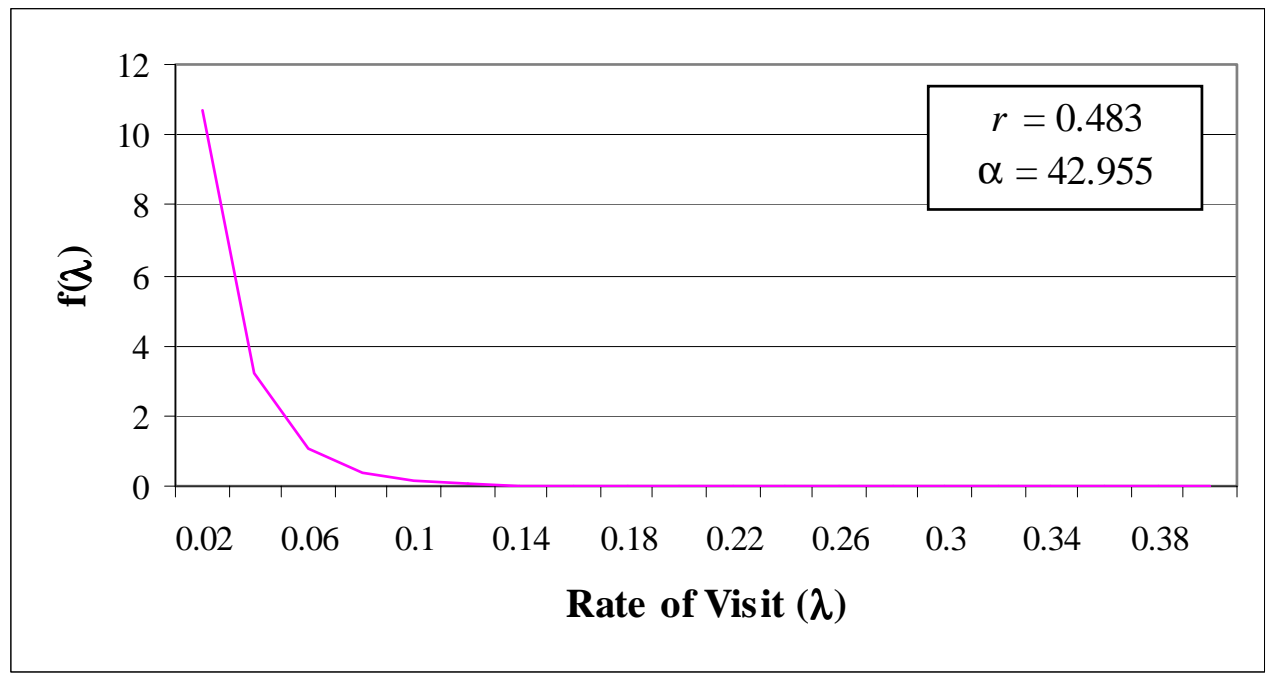


A principal reason for these high expected intervisit times is the fact that the stationary model does not allow shoppers to drop out and never return. As a result, a customer who has actually dropped out would be seen by the model as still being "alive," but having a very slow visiting rate, since she would not have yet returned to the store by the end of the observation period. The evolving visit model, however, allows for dropout (as well as evolving rates among visitors) and therefore provides more reasonable estimates of intervisit times.

In Table 2 we contrast the parameter estimates and fit statistics for the static EG model with those from our four-parameter model of evolving visiting behavior. Not only does the latter model fit the data better, but it also has more intuitively appealing results. While the basic shape of the gamma distribution for initial visit rates (shown in Figure 4a) may appear to be similar to that of the static EG model, it is less dominated by low-frequency shoppers, leading to a substantially lower mean intervisit time (52 days, $\mathrm{E}[\lambda]=0.019)$. Likewise, the median intervisit time shrinks to 167 days (median $\lambda=0.006$ ). These differences reflect the fact that dropout - or other types of evolution - can take place as the customer becomes more familiar with the site. 
Table 2. Model Results for Amazon

\begin{tabular}{|l|r|r|}
\hline & $\begin{array}{c}\text { Stationary EG } \\
\text { Model }\end{array}$ & \multicolumn{1}{c|}{$\begin{array}{c}\text { Evolving Visit } \\
\text { Model }\end{array}$} \\
\hline $\mathbf{R}$ & 0.483 & 0.324 \\
\hline $\boldsymbol{\alpha}$ & 42.955 & 16.857 \\
\hline $\mathbf{S}$ & & 2.299 \\
\hline $\boldsymbol{\beta}$ & & 2.304 \\
\hline & & \\
\hline LL & $-34,347.2$ & $-33,648.0$ \\
\hline No. of parameters & & 4 \\
\hline CAIC & $68,711.17$ & $67,296.0$ \\
\hline
\end{tabular}

Figure 4. Evolving Visit Model Distributions for Amazon Data

4a. Gamma Distribution of Initial Visiting Rates

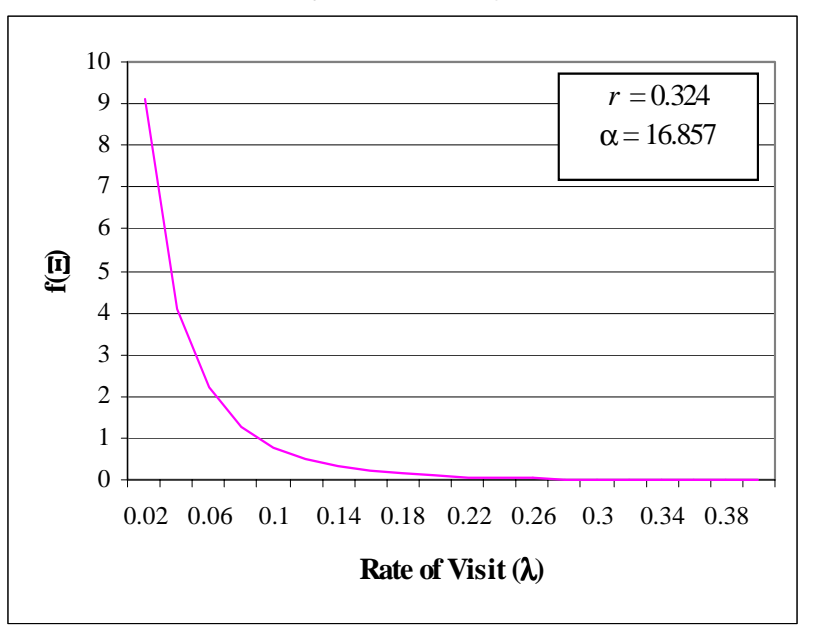

4b. Gamma Distribution of Updating Multiplier

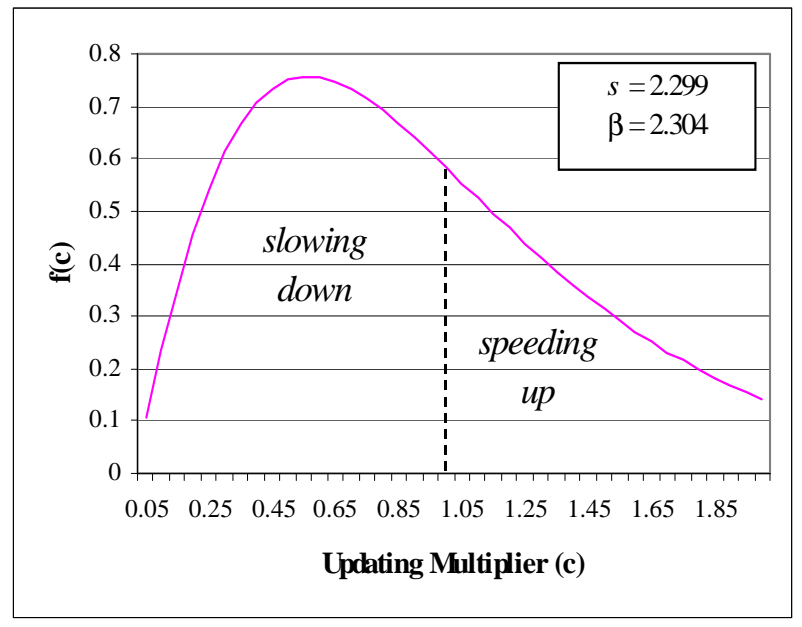

According to the evolutionary model, the mean update for any given visit $(s / \beta)$ is very close to one (0.998) suggesting, perhaps, that it is a fairly stationary process. However, a closer look at the distribution (see Figure $4 \mathrm{~b}$ ) shows that there is significant variance about this mean. Though the mean update is close to one, the distribution is quite skewed. With a median value of $c_{i j}=0.858$, shoppers tend to return to the store at slower rates from visit to visit. The implications 
of these results are in stark contrast to the measures summarized in Table 1 that implied increased visiting frequency over time.

Though other models have acknowledged the issue of nonstationarity, many of them have focused primarily on dropout (Eskin 1973, Kalwani and Silk 1980, Schmittlein, Morrison, and Colombo 1989). These models allow for individuals to make several purchases, become disenchanted, and never purchase again. To test if the evolving visit model is capturing evolving behavior over time in addition to a dropout phenomenon, we also estimated an exponentialgamma model with a dropout component similar to that specified by Eskin (1973) and Fader and Hardie (1999) $)^{4}$

In the EG model with dropout, the probability of visiting given that you are an active visitor is modeled as an exponential-gamma process. However, the probability of being an active visitor after the $j^{\text {th }}$ visit, $\pi_{j}$, is determined by the following:

$$
\pi_{j}=\phi\left(1-e^{-\theta j}\right)
$$

where $\phi$ is the long run probability of a customer remaining active, and $\theta$ is the rate at which the $\pi$ approaches this long run probability. Though the EG model with dropout provides a significant improvement in fit over the stationary EG model $(\mathrm{LL}=-33,804.7)$, it does not approach the performance of the evolving visit model which has the same number of parameters.

\footnotetext{
${ }^{4}$ When the Eskin model is estimated on this data set, log-likelihoods indicate a poorer fit (even with one more parameter) than the EV model.
} 
This suggests that the evolving visit model is capturing a phenomenon in addition to just dropout. $^{5}$

\section{Validation}

While we have discussed the fact that the evolving visit model fares well on a relative basis compared with various benchmark models, we have yet to show that it performs sufficiently well on an absolute basis. In this section, we validate the evolving visit model by examining the accuracy of longitudinal forecasts. Because the evolving visit model relies on an approximation (11) to specify and estimate the model, we need to perform simulations to generate data for tracking/forecasting purposes. This is a straightforward and computationally efficient task. For each iteration of the simulation, we create a simulated panel that matches the actual panel in terms of its size and the distribution of its initial visit times. We then generate a sequence of repeat visits using the parameter estimates from the model. This requires us to maintain a timevarying vector of $\lambda$ 's for each panelist, which starts with random draws from the initial $(r, \alpha)$ gamma distribution, and then gets updated using the $(s, \beta)$ gamma distribution after each simulated exponential arrival occurs. We continue this process until every simulated panelist gets past the tracking/forecasting horizon of interest to us. It is then a simple matter to count up the number of visits on a week-by-week basis for each iteration of the simulation. We then average across 1000 iterations to generate the tracking and forecasting plots. Using the MATLAB programming language, each of these iterations takes only a few seconds on a standard PC, and we see very consistent convergence properties after a few dozen iterations.

\footnotetext{
${ }^{5}$ We also tested several nested models that allowed for a constant update after every visit (i.e., equation 3), both with and without the dropout process. None of these models came close to the proposed evolving visit model in terms of fit or forecasting performance.
} 
Before creating the forecasts, we re-estimate both models (stationary and evolving EG) using only the first half (i.e., four months) of the dataset. (It is worth noting that the evolving model parameters are quite robust to this changing calibration period, while the stationary model has a noticeably higher visit rate over the shorter period - clear evidence of the slowdown discussed earlier). To generate the forecasts for the evolving visit model, we use the simulation procedure described above. For the stationary EG model, the expected number of repeat visits per week can be calculated directly as follows:

$$
E\left[\text { repeat visits } s_{w}\right]=N_{w} t\left(\frac{r}{\alpha}\right)
$$

where $N_{w}$ is the number of eligible repeat visitors in week $w$ and $t$ is the time period of interest, i.e., seven days in this case. Figure 5 shows cumulative forecasts as well as actual visits for the Amazon site.

Figure 5. Forecasts of Repeat Visits

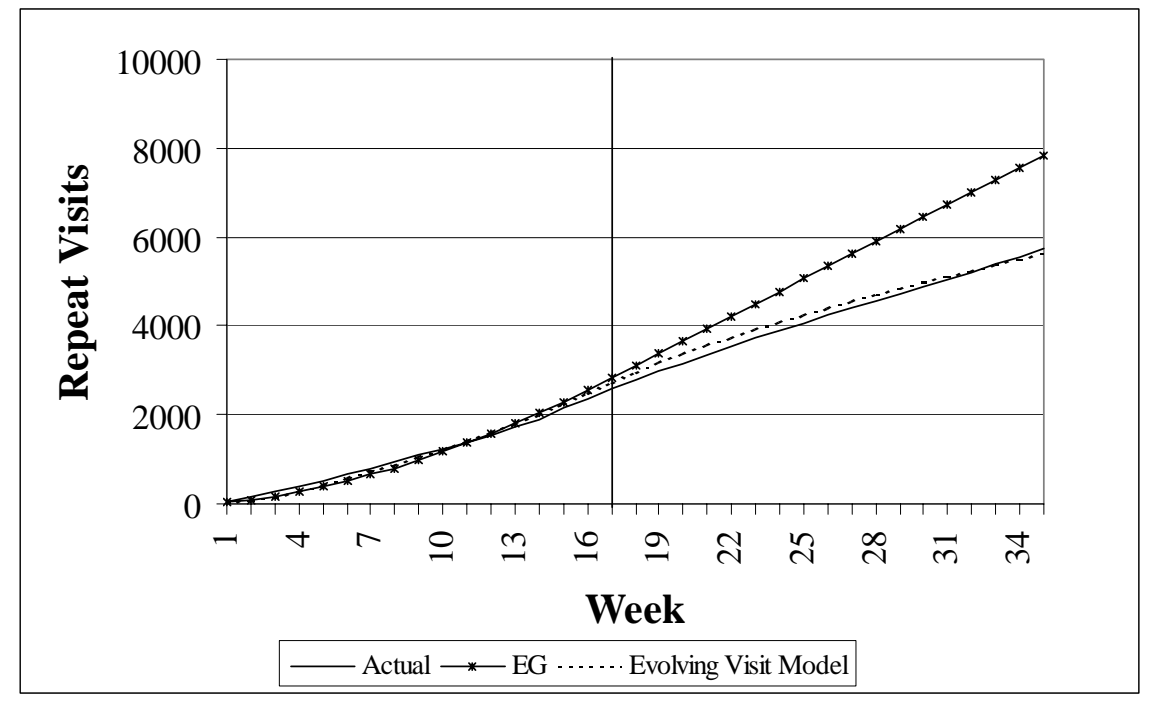

Both models seem to track the data quite well over the initial four-month calibration period. However, as we enter the forecasting period, the stationary EG model begins to diverge, 
ultimately overpredicting by $37 \%$ for Amazon at the end of the eight month period. It overestimates the number of visits per week as it does not recognize that shoppers are returning less frequently over time. The evolving visit model, however, forecasts quite accurately, well within $5 \%$ of the actual sales line throughout the forecast period. This is an impressive achievement and serves as a strong testimonial to the validity of the assumptions, structure, and parameter estimates associated with the proposed model.

\section{Results for CDNOW}

The same set of models and analyses were also applied to CDNOW data (results in Table 3). We see a remarkably similar set of patterns as in the case of Amazon. In moving from the static EG model to the evolving specification, we see significantly shorter intervisit times, since the latter model can accommodate customer dropout. We also see, once again, that the mean update is close to $1.0(0.991)$, but with a median of 0.837 , customer shopping frequency is more likely to decrease than increase after each visit. We emphasize once more that these results contradict the summary statistics from Table 1, which seemed to imply that shopping frequency is increasing from one visit cycle to the next.

Table 3. Model Results for CDNOW 


\begin{tabular}{|l|r|r|}
\hline & \multicolumn{1}{|c|}{$\begin{array}{c}\text { Stationary EG } \\
\text { Model }\end{array}$} & $\begin{array}{l}\text { Evolving Visit } \\
\text { Model }\end{array}$ \\
\hline $\mathbf{r}$ & 0.255 & 0.165 \\
\hline $\boldsymbol{\alpha}$ & 28.305 & 8.889 \\
\hline $\mathbf{s}$ & & 2.084 \\
\hline $\boldsymbol{\beta}$ & & 2.104 \\
\hline & & \\
\hline LL & $-9,459.6$ & $-9,120.7$ \\
\hline No. of parameters & & 4 \\
\hline CAIC & $18,934.1$ & $18,271.0$ \\
\hline
\end{tabular}

Other benchmark models (involving dropout and/or constant updates) proved once again to be vastly inferior to the evolving visit model. Finally, our forecast validation led to encouraging results with projected visits only $2 \%$ above the actual number at the end of the eight month period, compared to a $40 \%$ over-forecast for the stationary model. While we are very encouraged by these strong initial results, we are also surprised at the degree of similarity seen for these two sites. We certainly do not want to suggest that the specific patterns captured here will generalize to all online (or offline) retailers, but this should be ample motivation for future studies to find and describe a broader range of online visiting behavior.

\section{Visit Frequency and Evolution: Associations with Purchasing Behavior}

Studies of mall shopping behavior have shown that more frequent shoppers tend to be "recreational" shoppers - they are more involved and more motivated in the process and thus are more likely to impulse buy (Janiszewski 1998, Jarboe and McDaniel 1987, Roy 1994). From these studies and others, there is a wealth of evidence (theoretical and empirical) implying that 
more frequent shoppers are also more likely buyers at any given visit occasion. In this section, we explore this relationship between customers' visiting patterns and their purchasing propensities. We then extend the framework to incorporate (and separate out) the effects of evolving behavior on purchasing.

As an initial test of the traditional frequency-propensity hypothesis, we first calculate each panelist's expected rate of visit, $\lambda_{i}$, given the evolving visit model's estimated parameters and the panelists' observed behavior during the eight-month observation period. Using equations (12) and (13), we calculate each repeat visitor's mean rate of visit at the end of the observation period as $r\left(i, J_{i}\right) / \alpha\left(h, J_{i}\right)$. Across the 2098 repeat visitors to Amazon, the median expected visit rate at the end of our time period was 0.0349 or an intervisit time of 29 days.

Additionally, we calculate each panelist's purchasing propensity by dividing the number of visits during which a purchase occurred by the total number of visits made by that individual. The average conversion rate across the repeat visitors was 0.139 ; that is, almost $14 \%$ of the visits made by these customers were accompanied by a purchase. However, conversion rates differ for frequent shoppers, whom we define as customers with visiting rates greater than or equal to the median ( $\mathrm{N}=1062)$, versus infrequent shoppers, whom we define as customers with visiting rates less than the median $(\mathrm{N}=1036)$. Frequent visitors have significantly higher conversion rates, averaging $16.6 \%$ compared to an average across the infrequent visitors of $11.1 \%(t=6.04$, $p<0.001)^{6}$. These results confirm the hypothesis that frequent visitors tend to be more valuable

\footnotetext{
${ }^{6}$ To account for the non-normality of these proportions, we utilize a standard arc-sine transformation of the conversion rates for all of the statistical tests discussed in this section.
} 
customers since they are relatively more likely buyers, both on a percentage and an absolute basis.

However, the main objective of this paper is to capture - and capitalize upon - nonstationarity in individual's visiting behavior. Though the overall visit rates provide some information about the attractiveness of the visitor as a buyer, these rates change over time, and the nature of this change may have implications for the panelist's buying propensity. For example, new visitors may initially shop infrequently as they are unaccustomed to the environment. However, as they repeat visit, they begin to update their behavior. This evolving process may also be associated with greater purchasing propensity as it tends to lead to more involvement in the shopping process.

Therefore, in addition to segmenting panelists into frequent and infrequent visitors, we also characterize and segment panelists based on the extent of the behavioral evolution they have undergone during the observation period. For example, a frequent shopper who has always been a frequent shopper may be quite different from a frequent shopper who had recently evolved from being an infrequent shopper in the past.

To determine the extent of updating a panelist has undergone, we need to calculate a baseline rate of visit that would best capture their behavior if no evolution had taken place. Therefore, we calculate each individual's latent rate of visit given their observed behavior and the model results absent of any updating distribution (i.e., the value of $\lambda$ associated with a stationary EG model). 
The extent of updating for each panelist is the difference between their rate of visit as given by the nonstationary model and this baseline rate. ${ }^{7}$

The median update for repeat Amazon visitors is 0.000. A median split along this dimension divides shoppers into those who became more frequent visitors over time versus those who became less frequent visitors. We also see a difference in conversion rates $(\mathrm{CR})$ along this dimension: those who increased their rate of visit were more likely to buy $(\mathrm{N}=1056, \mathrm{CR}=15.1 \%)$ than those who decreased their rate of visit $(\mathrm{N}=1042, \mathrm{CR}=12.7 \%)$. Once again, this difference is highly significant $(t=2.68, \mathrm{p}=0.007)$, suggesting that the degree of evolution is indeed related to purchase propensity.

After seeing these two strong effects, a natural question is whether each one is still present when both are taken into account simultaneously. Table 4 examines the issue by dividing repeat visitors along both dimensions into four cells, using the same median splits as before. It is interesting to note that the number of visitors in each cell is quite balanced, indicating that there is not a dominant association between frequency and updating. In other words, for every household that started with a slow visit rate and sped up towards the end of the model calibration period, there is a corresponding household that started with a very fast visit rate, but slowed down to roughly the same level by the end of the eight-month period.

Table 4. Amazon's Conversion Rates

\footnotetext{
${ }^{7}$ There is no significant difference in the relative position of each household in terms of its extent of evolution when the change in visiting rates is measured as an absolute difference versus a percentage change.
} 


\begin{tabular}{|c|c|c|}
\hline median $=0.0349$ & Decreasing Frequency & Increasing Frequency \\
\hline Infrequent Visitors & $\mathrm{CR}=10.9 \%(\mathrm{~N}=526)$ & $\mathrm{CR}=1 \frac{\text { CELL 2 }}{1.3 \%(\mathrm{~N}=510)}$ \\
\hline Frequent Visitors & $\frac{\text { CELL 3 }}{\mathrm{CR}}=14.6 \%(\mathrm{~N}=516)$ & $\mathrm{CR}=18.6 \%(\mathrm{~N}=546)$ \\
\hline
\end{tabular}

An ANOVA on these data confirm that both main effects remain highly significant: $F_{1,2094}=$ $35.765(p<0.001)$ for high vs. low frequency, and $F_{1,2094}=6.473(p=0.011)$ for increasing vs. decreasing frequency. Furthermore, a strong interaction $\left(F_{1,2094}=5.035, p=0.025\right)$ emerged as well, and its presence is easily seen in Table 4. For infrequent visitors (top row), there is no meaningful difference in conversion rates, regardless of the nature of the household's updates over time. But for frequent visitors, the purchase-to-visit rate is considerably higher for those who have experienced increasing frequency. The households in the lower right cell are particularly conspicuous, with a conversion rate nearly $40 \%$ higher than the rest of the panel. This is clearly a very attractive group of repeat buyers. ${ }^{8}$

Table 5 presents the same analysis for the 581 households that made at least one repeat visit to CDNOW. The patterns are remarkably similar to those seen for Amazon, with the exception of smaller sample sizes and lower conversion rates. The ANOVA model reveals significant main effects $\left(F_{1,577}=4.044, p=0.045\right.$ for frequency, and $F_{1,577}=8.810, p=0.003$ for updating $)$, with a very strong interaction $\left(F_{1,577}=6.405, p=0.012\right)$ once again highlighting the unique nature of those households that have accelerated their visiting behavior to a relatively high rate over the course of the eight-month data collection period. The conversion rate for the households in this

\footnotetext{
${ }^{8}$ In addition to this ANOVA conducted on the two dichotomous variables discussed here, we also examined equivalent regression models on the household-level data. The results are quite similar across the two datasets.
} 
cell is over $60 \%$ higher than that of the three cells combined. While this translates to only 3 percentage points on an absolute basis, this represents a very significant improvement in an industry that is just becoming aware of the critical importance of this single statistic as the most useful indicator of an online retailer's performance and future prospects (Gurley 2000).

Table 5. CDNOW's Conversion Rates

\begin{tabular}{|c|c|c|}
\hline median $=0.0431$ & Decreasing Frequency & Increasing Frequency \\
\hline Infrequent Visitors & $\mathrm{CR}=3.8 \%(\mathrm{~N}=129)$ & $\mathrm{CR}=5.7 \%(\mathrm{~N}=161)$ \\
\hline Frequent Visitors & $\mathrm{CR}=4 . \frac{\text { CELL 3 }}{.0 \%(\mathrm{~N}=160)}$ & 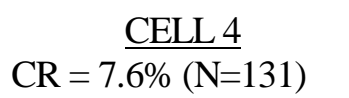 \\
\hline
\end{tabular}

Taken together, the analyses for these two leading online retailers suggest not only that frequent visitors are more likely buyers, but also that a more refined segmentation of visitors that incorporates changes in visiting behavior can identify an even more valuable segment of customers to target. This is a new and important result, worthy of management attention and further research.

\section{Discussion and Conclusions}

Many skeptics claim that the Internet is nothing more than a new distribution channel, and thus it should not change the way we examine customer behavior. While this may be true in certain respects, this paper highlights some of the uniquely different research perspectives that we gain from examining clickstream data. Thanks to rich new sources of data (such as Media Metrix), we can now examine behavioral phenomena that would be impossible to study using more traditional sources, such as grocery store scanner data. 
The detailed, disaggregate data available to us make it possible to study the evolution of visit behavior at a retail site. The model developed here is not tailored specifically to online stores, although it might be hard to obtain the necessary data to estimate this model for a "bricks-andmortar" retailer. For instance, many traditional retailers use some sort of tracking mechanism, e.g., a loyalty card, to capture the timing of purchases at the store, but it is hard for them to capture visits that do not involve a purchase.

We posit a behaviorally plausible - and highly parsimonious - model that allows visiting behavior to evolve gradually over time, although it also allows for more abrupt changes, such as permanent dropout from the site. And indeed, our empirical analysis reveals the fact that the average update in household visiting rates is a multiplier close to 1.0, but there is significant spread around this value. Additionally, the manner in which we implement this updating scheme - a gamma distribution to capture the different values of these multipliers - is a new methodological contribution, which merits consideration for other types of non-stationary modeling contexts.

Use of the model reveals that individual-level behavior patterns appear to contradict the perspective that one would obtain from examining the aggregate data alone. Specifically, the aggregate data seem to indicate an acceleration of visiting behavior at each of two leading ecommerce sites, yet our model parameters suggest that the typical shopper is experiencing a gradual slowdown in her visiting rate over time. The difference here is that an increasing number of new visitors are coming to each site over time, masking the slowdown that may be occurring 
for many experienced visitors. This effect could have dramatic implications for managers who neglect to examine their data at a sufficiently fine level of disaggregation.

Beyond the intuitive appeal of the model specification and its estimated parameters, we also show that it has excellent validity from an out-of-sample forecasting perspective. For both retail sites, the model tracks future visiting patterns extremely well, remaining within 5\% of the actual data over the entire duration of a four-month holdout period. While this model was not constructed with forecasting in mind as a principal objective, this result certainly speaks well about its overall versatility.

Perhaps the most dramatic demonstration of the model's validity and usefulness is its ability to delineate highly significant differences in purchasing behavior across shoppers. There is a significant amount of past literature suggesting that customers who visit a particular store frequently also tend to buy something during a relatively high proportion of those shopping trips. We provide strong confirming evidence of this hypothesis. But the evolutionary nature of our model allows us to test an equally compelling complementary hypothesis: people who experience increases in their visiting rates over time are more likely to purchase something at any given visit than those who are slowing down.

Both sites provide solid support for this new hypothesis, but also exhibit a powerful interaction that combines both of these effects. Specifically, panelists who combine high frequency with an upwards evolutionary trend in visiting behavior have dramatically higher conversion rates than all other panelists. As noted above and elsewhere (e.g., Forrester 1999) measuring and managing 
conversion rates is becoming increasingly crucial to e-commerce executives, so this is an important finding that merits additional investigation in later research.

\section{Limitations and Future Research}

Since this paper is among the first attempts to carefully examine online visiting behavior using clickstream data, we have deliberately kept the model as clear and simple as possible in order to highlight the chief phenomena that we have observed in these datasets. However, one limitation is the fact that the data does not reveal when each customer first started visiting each e-tailer. As a result, the model is only able to provide a description of customer visiting rates and how they are changing during the data period being examined. In time, as all potential customers have adopted and become accustomed to the online store environment, perhaps no evolution will be detected. However, the EV model presented in this paper will allow e-tailers to monitor trends until that time comes and also know when that time has arrived.

But as the types of data and methods employed here become more commonplace, we can see several extensions to the model that may be worth pursuing. Because we have been emphasizing the importance of evolution in a new marketplace (such as online sales of books and CD's) we have paid little attention to the fact that these markets might eventually shift towards a more steady-state nature, i.e., with updates occurring less frequently and with smaller magnitudes. It is unlikely that the same distribution of updating multipliers $\left(c_{h j}\right)$ will stay in place over a long period of time. Perhaps this distribution starts to collapse towards a spike at 1.0 as the market matures. The excellent performance of our holdout forecasts does not seem to indicate any such 
pattern in our datasets, but as our observation window extends to several years' worth of data in the future, we might see more benefits from such a specification.

Another way of improving on the $c_{i j}$ distribution might be to let these multipliers vary more systematically across customers and visits. Rather than assuming, as we do now, that each update is an independent draw from the same distribution of multipliers, we can allow the draws to be linked over time at the household level, and also allow the shape of the distribution to vary over time and across households. These extensions would require the use of computationally intensive hierarchical Bayes estimation procedures, which would then also enable the inclusion of other features, such as allowing for a correlation structure between the set of visit rate parameters and the update multipliers. But all of these extensions are well beyond the scope of this initial analysis.

Beyond these methodological issues on our "to-do" list, it is important to acknowledge the need for further process-oriented research to better explain and extend the psychological mechanisms underlying our findings concerning the relationships between conversion rates and visit dynamics. While there is ample theoretical reasoning behind the well-established frequency hypothesis, it would be useful to establish an equally solid base of explanations and controlled experimental evidence for the effect of positive vs. negative evolution, as well as the substantial interaction effect we have observed.

Finally, our brief examination of conversion rates suggests that there is a need for modeling efforts that are more focused on this phenomenon by itself. While we have allowed visit 
behavior to evolve in our model, we have treated conversion rates as a purely static summary

measure. In reality, however, the relationship between visits and purchases is likely to go

through its own type of evolution. Once we have a complete understanding of the dynamic visitpurchase process, we can combine such a model with the present "visit only" model to obtain a complete picture of online buying behavior. 


\section{REFERENCES}

Alba, Joseph W. and J. Wesley Hutchinson (1987), "Dimensions of Consumer Expertise," Journal of Consumer Research, 13 (March), 411-454.

Bellinger, Danny N., Dan H. Robertson, and Elizabeth C. Hirschman (1978), "Impulse Buying Varies by Product," Journal of Advertising Research, 18 (December), 15-18.

Bronnenberg, Bart J., Vijay Mahajan, and Wilfried R. Vanhonacker (2000), "The Emergence of Market Structure in New Repeat-Purchase Categories: The Interplay of Market Share and Retailer Distribution," Journal of Marketing Research, 37 (February), 16-31.

Cooperstein, David M., Kate Delhagen, Alexander Aber, and Kip Levin (1999), "Making Net Shoppers Loyal," The Forrester Report (June).

Demers, Elizabeth and Baruch Lev (2000), “A Rude Awakening: Internet Shakeout in 2000," September, Working Paper.

Eskin, Gerald J. (1973), "Dynamic Forecasts of New Product Demand Using a Depth of Repeat Model," Journal of Marketing Research, 10 (May), 115-129.

Fader, Peter S. and Bruce G.S. Hardie (1999), "Investigating the Properties of the Eskin/Kalwani \& Silk Model of Repeat Buying for New Products," in Lutz Hildebrandt, Dirk Annacker, and Daniel Klapper (eds.), Marketing and Competition in the Information Age, Proceedings of the 28th EMAC Conference, May 11-14, Berlin: Humboldt University.

Fader, Peter S. and James M Lattin (1993), "Accounting for Heterogeneity and Nonstationarity in a Cross-Sectional Model of Consumer Purchase Behavior, Marketing Science, 12 (3), 304-317.

Gurley, J. William (2000), “The one Internet metric that really matters," Fortune. 141(5): 392.

Howard, R.A.(1965), “Dynamic Inference,” Operations Research, Vol. 13 (2), 712-733.

Janiszewski, Chris (1998), “The Influence of Display Characteristics on Visual Exploratory Search Behavior," Journal of Consumer Research, 25 (3), 290-301.

Jarboe, Glen R. and Carl D. McDaniel. (1987). "A Profile of Browsers in Regional Shopping Malls," Journal of the Academy of Marketing Science, 15 (Spring): 46-53.

Johnson, Eric and J. Edward Russo (1984), "Product Familiarity and Learning New Information," Journal of Consumer Research, 11 (June), 542-550.

Kalwani, Manohar and Alvin J. Silk (1980), "Structure of Repeat Buying for New Packaged Goods," Journal of Marketing Research, 17 (August), 316-322, 
Kendall, Maurice G. and Alan Stuart (1977), The Advanced Theory of Statistics, $3^{\text {rd }}$ edition, vol. 2, New York: Hafner.

Morrison, Donald G. and David C. Schmittlein (1988), "Generalizing the NBD Model for Customer Purchases: What Are the Implications and Is It Worth the Effort?" Journal of Business \& Economic Statistics, 6 (2), 145-159.

Park, C. Whan, Easwar S. Iyer, and Daniel C. Smith (1989), "The Effects of Situational Factors on InStore Grocery Shopping Behavior: The Role of Store Environment and Time Available for Shopping," Journal of Consumer Research, 15 (4), 422-433.

Reinartz, Werner J. and V. Kumar (2000), "On the Profitability of Long-Life Customers in a Noncontractual Setting: An Empirical Investigation and Implications for Marketing," Journal of Marketing, 64 (4), 17-35.

Roy, Abhik (1994), “Correlates of Mall Visit Frequency,” Journal of Retailing, 70 (2), 139-161.

Sabavala, Darius J. and Donald G. Morrison (1981), "A Nonstationary Model of Binary Choice Applied to Media Exposure," Management Science, 27 (6), 637-657.

Schmittlein, David C., Donald G. Morrison, and Richard Colombo (1987), "Counting Your Customers: Who Are They and What Will They Do Next?" Management Science, 33, 1-24. 


\section{APPENDIX A. Moment-Matching Approximation of the Product of Two Gamma Distributions}

If $x$ and $y$ are two gamma distributed random variables,

$\mathrm{x} \sim \operatorname{Gamma}(\mathrm{r}, \mathrm{a})$

$\mathrm{y} \sim \operatorname{Gamma}(\mathrm{s}, \mathrm{b})$

then the product, $z=x y$, can be assumed to be a gamma distributed random variable

$z \sim \operatorname{Gamma}(R, A)$

with shape and scale parameters, $R$ and $A$, such that the first two raw moments of the $z$-distribution is the product of the moments of the $x$ - and $y$-distributions.

$$
\begin{array}{ll}
m_{1}{ }^{x}=\frac{r}{\alpha} & m_{2}{ }^{x}=\frac{r(r+1)}{\alpha^{2}} \\
m_{1}{ }^{y}=\frac{s}{\beta} & m_{2}{ }^{y}=\frac{s(s+1)}{\beta^{2}} \\
m_{2}{ }^{z}=m_{1}{ }^{x} \cdot m_{1}{ }^{y}=\frac{r s}{\alpha \beta} & m_{2}{ }^{z}=m_{2}{ }^{x} \cdot m_{2}{ }^{y}=\frac{r(r+1) s(s+1)}{\alpha^{2} \beta^{2}}
\end{array}
$$

Since the first moment of the $z$-distribution, $m_{1}{ }^{\mathrm{z}}$, is $R / A$ and the second moment, $m_{2}{ }^{\mathrm{z}}$, is $R(R+1) / A^{2}$, we can solve for $R$ and $A$ with the following two equations:

$$
\frac{R}{A}=\frac{r s}{\alpha \beta} \quad \frac{R(R+1)}{A^{2}}=\frac{r(r+1) s(s+1)}{\alpha^{2} \beta^{2}}
$$

Therefore, the gamma distribution describing the product of two independently distributed gamma random variables has shape and scale parameters that can be calculated from the parameters of the multiplying distributions.

$$
R=\frac{r s}{(r+1)(s+1)-r s} \quad A=\frac{\alpha \beta}{(r+1)(s+1)-r s}
$$

with Bayesian updating after observing one arrival at time $t$...

$$
R=\frac{(r+1) s}{(r+2)(s+1)-(r+1) s} \quad A=\frac{(\alpha+t) \beta}{(r+2)(s+1)-(r+1) s}
$$

